\title{
Movement-Aware Relay Selection for Delay-Tolerant Information Dissemination in Wildlife Tracking and Monitoring Applications
}

\author{
Yuhui Yao, Yan Sun, Chris Phillips, and Yue Cao, Member, IEEE
}

\begin{abstract}
As a promising use-case of the Internet of Things (IoT), wildlife tracking and monitoring applications greatly benefit the ecology-related research both commercially and scientifically. In literature, a Forward-Wait-Deliver strategy has been researched to facilitate energy-efficient dissemination of delaytolerant information, which penitentially contributes to long-term tracking and monitoring. However, this strategy is not directly applicable for wildlife tracking and monitoring applications, as the movement trajectory of animals cannot be precisely predicted for relay selection. To this end, further studies are required to utilise partially predictable mobility based on more generalised navigational information such as the movement direction. In this paper, the feasible exploitation of directional movement in pathunconstrained mobility is investigated for strategic forwarding. Our proposal is an advance to the state-of-the-art because the directional correlation of destination movement is considered to dynamically exploit the node mobility for the optimal selection of a stationary relay. Simulation results show that higher delivery utility can be achieved by the proposed fuzzy path model compared with a forwarding scheme without contact prediction or one based on linear trajectory model.
\end{abstract}

Index Terms-directional correlation, movement estimation, contact prediction

\section{INTRODUCTION}

As a promising use-case of the Internet of Things (IoT), wildlife tracking and monitoring applications greatly benefit ecology-related research both commercially and scientifically [1]. With the deployment of numerous smart sensors/motes, remote close observation can be achieved on wild animals (e.g. deer [2], birds [3], fish [4], etc.) while unattended actions can be taken in a timely manner by automatic actuators (e.g. robotic drones [5]). Further accelerated by emerging technologies such as cloud computing [6] and M2M (Machineto-Machine) communication [7], these IoT applications will certainly provide an unprecedented panoramic view of the natural world.

Due to changing environments and requirements, it is a common need to reconfigure sensors and actuators (together

Manuscript accepted April 24, 2018. (Corresponding author: Yue Cao)

Y. Yao, Y. Sun, and C. Phillips are with the Department of Electronic Engineering and Computer Science, Queen Mary University of London, London E1 4NS, U.K. (e-mail: yuhui.yao@qmul.ac.uk; yan.sun@qmul.ac.uk; chris.i.phillips@qmul.ac.uk)

Y. Cao is with the Department of Computer and Information Sciences, Northumbria University, Newcastle upon Tyne NE1 8ST, U.K. (e-mail: yue.cao@northumbria.ac.uk)

Copyright (c) 2012 IEEE. Personal use of this material is permitted. However, permission to use this material for any other purposes must be obtained from the IEEE by sending a request to pubs-permissions@ieee.org. referred as nodes in the remainder of this paper) from the sink (referred to as the gateway node which connects the rest of nodes with outside networks) [8]. To disseminate this configuration information (i.e. commands and updates), wireless multi-hop forwarding (called ad hoc networking) is a cost-effective solution when there is difficulty in directly reaching the target node, for instance, cases where there is limited communication capability or available infrastructure as is typical in the wild (such as forests and underwater). Via routes established between intermediate nodes, the whole network becomes reachable by the sink without relying on extra communication infrastructure (as shown in Figure 1). Nevertheless, because the forwarding behaviour is performed by resource-constrained wireless nodes, the energy efficiency of dissemination is a crucial issue to support long-term (months or years) tracking and monitoring.

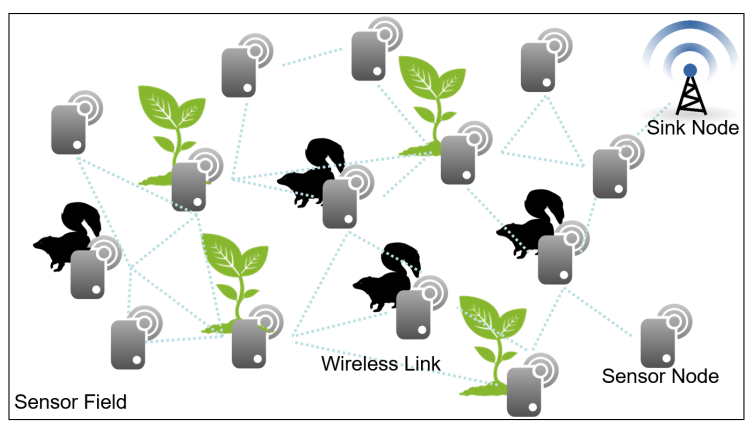

Fig. 1. Scenario Example of Wildlife Tracking and Monitoring Applications

In literature, a Forward-Wait-Deliver (FWD) strategy has been adopted (such as [9]-[11]) to facilitate energy-efficient dissemination of delay-tolerant information. Instead of immediate delivery to the mobile destination, packets are forwarded to a relay ${ }^{1}$ in advance, waiting for later delivery when the destination node is directly contactable. Given the movement trajectory reported by destination nodes (e.g. the vehicular navigation system), the FWD strategy can make full use of the possible delay budget to find an optimal forwarding route (usually by employing fewer hops as shown in Figure 2), which is beneficial for reducing energy consumption and prolonging system lifetime. To make the problem more tractable, each delivery is assumed to be single-copied in this paper (i.e. no redundant copies are used to improve the possibility

\footnotetext{
${ }^{1}$ the relay is selected from a set of nodes who are assumed to be temporarily or permanently stationary
} 
of successful delivery) while a multi-copied scheme could be researched in future work.

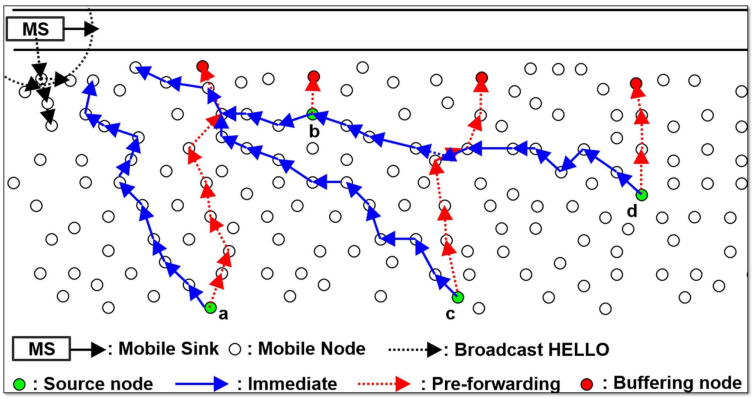

Fig. 2. Reduction of Forwarding Hops by Forward-Wait-Deliver Strategy [9]

With regard to the delay-tolerant configuration information (e.g. pre-planned updates), the FWD strategy has the potential to bring the gain of dissemination efficiency. However, wildlife tracking and monitoring applications place high demand on deploying devices (attached or implanted) to animals, so the movement trajectory of these mobile nodes becomes less possible to be precisely predicted for relay selection. Meanwhile, animals usually move with a certain purpose (such as searching or migrating [12]) and therefore their movement reflects more-or-less predictability. To this end, further studies are required to utilise partially predictable mobility for adopting the FWD strategy based on more generalised navigational information such as the movement direction.

In this paper, the feasible exploitation of directional move-

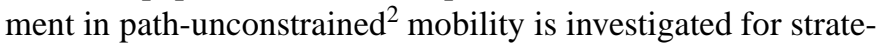
gic forwarding. The premise of our research is straightforward: a degree of persistence in the future direction of travel is expected on the basis of the current movement direction of a mobile node. As a consequence, there is a fair chance that a stationary node lying along this path will encounter the mobile node. Given adequate budget of delivery delay and enough confidence of movement persistence (i.e. the tendency to keep moving towards), the risk associated with opportunistic delivery probably can be balanced by the desire to reduce the number of forwarding hops. To the best of our knowledge, no existing research has provided such a quantitative analysis for strategic packet forwarding based on the comprehensive consideration of node mobility, network topology, and delivery requirements.

The main contribution of our research is a systematic framework for analytical modelling of relay selection.

- Our proposal advances the state-of-the-art by considering the directional correlation of destination movement to dynamically exploit the node mobility for optimal selection of a stationary relay.

- A novel fuzzy path model is proposed for movement estimation, where the influence of directional correlation is quantified by the standard deviation of turning angles.

- By modelling of directional correlation, the proposed model provides more flexible estimation of directional

\footnotetext{
${ }^{2}$ mobile individuals independently move in relatively unobstructed environment (such as a forest or ocean)
}

movement within a path-unconstrained scenario where navigation systems are unavailable.

The rest of this paper is organized as follows. Section II introduces the related work to further demonstrate the research motivation and novelty. Section III and IV provide the detailed system design and theoretical analysis. In Section V, simulation results are shown and the performance is discussed. The paper is finally concluded in Section VI.

\section{RELATED WORK}

Our study concerns the exploitation of node mobility for packet delivery. Among the state-of-the-art forwarding schemes, six types of mobility description for contact prediction are classified from the literature (as summarised in Table I). For these existing contact prediction schemes, the underlying movement estimation models are first discussed. Next, related studies on mobility modelling are reviewed, particularly in regard to the description and estimation of directional movement. Finally a summary concludes the research novelty.

\section{A. Modelling of Predictable Contact}

For the ease of contact prediction, sufficient knowledge of node movement is usually assumed according to the first three types of mobility description listed in Table I. For the Type-I class, a complete travel plan is assumed to be known in advance and therefore future contactable locations can be directly predicted. Knowledge of predefined waypoints usually relies on access to the navigation system, for example to obtain a report of route information from vehicles, as assumed in [10] [11]. For the Type-II class, although the knowledge of whole movement path is not required, strict periodicity is assumed in the contact interval (such as in [13] [14]). For the TypeIII class, the node mobility assumptions are further relaxed as a stochastic process. For instance, the invariant properties of the inter-contact time are discovered between vehicles and roadside units in [15] [16]. However, stochastic properties can vary with cases and their analysis requires traces recorded over a long term and/or of a large quantity.

When the mobility knowledge is limited, the estimation of node movement plays an important role in contact prediction. For the last three types listed in Table I, the latest location of the mobile node is taken as the reference point and the contact is predicted by estimating the node movement. For the Type-IV class, a maximum (or average) movement speed is estimated for the mobile node, so a circular movement range can be drawn to predict contact (as in [17] [18]), where the radius depends on the delay since the location report. For the Type$\mathrm{V}$ class, with additional knowledge on movement direction, the location of future contact can be expected given the delay (such as in [19] [9]). However, the speed and direction are not enough to differentiate heterogeneous mobility, which limits the contact prediction based on the Type-IV/V mobility description. For the Type-VI class, a diffusion constant is introduced by [20] based on the exponent parameter of the step-length distribution in Lévy walk model. Compared with the movement speed, the diffusion constant is a better 
TABLE I

Classification of Mobility Description for Contact Prediction with Underlying Movement Estimation

\begin{tabular}{|c|c|c|c|}
\hline Type & Mobility Description & Movement Estimation & Contact Prediction \\
\hline I & Complete travel plan & Defined waypoint model & Contactable locations (and the waiting time before contact) \\
\hline II & Explicit return period & Cyclic visit model & Contact interval (with/without uncertainty) \\
\hline III & Historical traces collection & Stochastic arrival model & Distribution of inter-contact time (and contact duration) \\
\hline IV & Value of estimated speed & Circular range model & Contactable area given delay \\
\hline V & Estimated speed with direction & Linear trajectory model & Expected contact location given delay \\
\hline VI & The diffusion exponent & Diffusive behaviour model & Contact likelihood given relative location \\
\hline
\end{tabular}

characterisation of diffusive behaviour, as the randomness of step length is taken into consideration. Nevertheless, to the best of our knowledge there remains no prior work that considers differentiation of directional movement for contact prediction.

\section{B. Modelling of Directional Movement}

Unlike the completely unpredictable movement imitated by memoryless random mobility, mobile nodes tend to move smoothly in many cases and sharp turns rarely occur [21]. To reflect this directional movement behaviour, correlated motion patterns are researched in the literature. The existing pattens for directional correlation can be roughly divided into two branches: velocity-based or step-based. For the first branch, correlation patterns focus on the gradual change of velocity which leads to the persistence in movement direction. In [22], the degree of temporal correlation is governed by a memory level parameter, but difficulty arises from controllable correlation of movement direction because the velocity component in each spatial dimension is independently modelled as a Gauss-Markov process. In [23], despite the modelling of accelerations, the probability in choosing movement direction is basically isotropic because the authors purely focus on dividing changes into several small increments. In contrast, the correlation patterns in the second branch are based the characterisation of step direction. In [24] [25], the directional correlation between random steps is investigated but their research is restricted to an n-dimensional lattice or grid. In [26], the directional preference of random step is described by a probability distribution model, but an additional assumption is required for fixing a bias of movement direction. In [27], the angle of maximum turn is introduced to bound the Random Turning Angle (RTA), which results in a directional correlation between steps. However, the influence of RTA in movement is not analysed by [27] or any later reference, as their focus is on the network simulation rather than movement analysis.

From the biophysics-related fields, further studies on the RTA are found concerning the Correlated Random Walk (CRW) model [28]. In [29], circular distribution functions are employed as the model of RTA, which is theoretically rigorous but introduces additionally complexity. In [30], both the angle of maximum turn and the Standard Deviation of the Turning Angles (SDTA) are considered to characterise the probability distribution of RTA. In [31], the probability distribution model of RTA is linked to the diffusion degree to analyse the movement of animals. For the aforementioned research on CRW, the distance-based metrics, such as the mean square displacement, have been commonly analysed and formulated. However, the calculative method to estimate the displacement angle based on the distribution characteristics of
RTA cannot be found from the literature. Furthermore, to the best of our knowledge, none of existing works include a formal discussion regarding a truncated distribution model for RTA.

\section{Summary}

The above literature review suggests that the state-of-the-art (as classified in Table I) is insufficient to reasonably predict contact caused by directional movement. By introducing directional correlation as an index of node mobility, predictable contact can be extended to facilitate strategic forwarding, although problems remain unsolved in terms of characterisation and exploitation. From an analytical modelling perspective, our proposal is described in Section III with further analysis provided in Section IV and V.

\section{System ModeL}

In the previous sections, the role of mobility analysis and how it can be exploited has been considered. To overcome the remaining challenge of exploiting directional movement in path-unconstrained mobility, an analytical framework (with key notations listed in Table II) is proposed in this section and is elaborated from three aspects. First, the correlation pattern of node mobility is considered to represent directional movement. Second, based on the mobility model, contact opportunities are mathematically analysed as a stochastic process. Finally, the delivery utility is defined and analysed.

\section{A. Mobility Model}

Devising a suitable mobility model is the first step in creating a successful dynamic delivery mechanism. Without loss of generality, we consider a mobile target moves within a two-dimensional space $\mathbb{R}^{2}$.

The fluctuations of movement speed are assumed to be negligible compared with the mean value, so that the average speed (denoted by $V$ ) is considered to be a constant as

$$
V=v(t)-\Delta v(t) \approx v(t)
$$

where $v(t)$ denotes the movement speed at time point $t$ and $\Delta v(t)$ denotes the speed variation from the average at time point $t$.

The movement direction (denoted by $\theta \in[-\pi, \pi]$ ) is assumed to be fixed during a step which is a constant period defined as

$$
T=t_{i+1}-t_{i}
$$

where $i \in \mathbb{N}$ denotes the discrete step number corresponding to the time point $t_{i}$. 
TABLE II

LIST OF KEY NOTATIONS

\begin{tabular}{|c|l|}
\hline Notation & Explanation \\
\hline$V$ & the average movement speed \\
\hline$T$ & the duration of a step period \\
\hline$\Theta$ & the movement direction \\
\hline$\Theta$ & the random turning angle between successive steps \\
\hline$\lambda_{i}$ & the movement distance during step $i$ \\
\hline$\omega_{i}$ & the movement direction during step $i$ \\
\hline$\vec{e}_{i}$ & the displacement vector after $i$ steps \\
\hline$R$ & the radius of contact range \\
\hline$n_{j}$ & a stationary node \\
\hline $\mathcal{N}$ & the whole set of stationary nodes \\
\hline$d_{j}$ & the distance from $n_{j}$ to the mobile target \\
\hline$\vec{e}_{j}$ & the relative position coordinates of $n_{j}$ \\
\hline$H_{j}$ & the forwarding hops from the gateway node to $n_{j}$ \\
\hline$m_{k}$ & a task of delivery from the gateway to a mobile destination \\
\hline $\mathcal{M}$ & the whole set of delivery tasks \\
\hline$M$ & the total number of delivery tasks \\
\hline$B_{k}$ & the delay budget of $m_{k}$ \\
\hline$h_{k}$ & the minimum hop number for $m_{k}$ \\
\hline$c_{k}$ & the forwarding cost for $m_{k}$ \\
\hline$s_{k}$ & the expected satisfactory degree of delivery \\
\hline$\tau, \tau_{k}$ & a upper bound of step number (given $B_{k}$ ) \\
\hline$\mu, \mu_{\tau}$ & the certainty degree of contact (within step bound $\tau$ ) \\
\hline$\eta$ & a threshold of certainty degree \\
\hline $\mathcal{N}_{\tau, \eta}$ & $\begin{array}{l}\text { the subset of stationary nodes considered as the movement } \\
\text { path of the mobile target given } \tau \text { and } \eta\end{array}$ \\
\hline $\mathcal{N}_{k}$ & the qualified candidates of stationary nodes for $m_{k}$ \\
\hline$u$ & the delivery utility as the ratio of overall satisfactory degree \\
to the overall forwarding cost \\
\hline
\end{tabular}

Between two successive steps, the movement direction is updated by an angle of random turning

$$
\Theta_{i}=\theta_{i}-\theta_{i-1}
$$

where $\theta_{i}$ denotes the movement direction during time step $i$, and $\Theta_{i} \in[-\pi, \pi]$ denotes the random turning angle between step $i-1$ and step $i$.

For the movement made during the $i$ time step, $\lambda_{i}$ denotes the step movement distance and $\omega_{i}$ denotes the step movement direction. Based on equations (1)(2) and (3), the step movement distance and direction can be derived as

$$
\begin{gathered}
\lambda_{i}=V \cdot T \\
\omega_{i}=\theta_{i}=\theta_{i-1}+\Theta_{i}
\end{gathered}
$$

Given an initial movement direction (denoted by $\theta_{0}$ ), equation (5) can be further rewritten as

$$
\omega_{i}=\theta_{0}+\sum_{\zeta=1}^{i} \Theta_{\zeta}
$$

Consequently, the real-time movement of a mobile target can be specified given the initial direction and the probability distribution of the random turning angles. Under such a mobility model, the persistence of the initial movement direction progressively diminishes over successive time steps, which will be further discussed in the Section IV.

\section{B. Contact Model}

Having described the mobility model, we now turn our attention to the modelling of contact. A traditional model of unit disk [32] is considered where there is a constant radius of contact range (denoted by $R$ ). Let $\mathcal{N}$ denote the whole set of stationary nodes, then a stationary node (denoted by $n_{j}$ ) is contactable by the mobile object if only if

$$
d_{j}(i) \leqslant R
$$

where $d_{j}(i)$ denotes the distance between $n_{j}$ and the mobile target as a function of step number $i$.

The certainty degree of contact (denoted by $\mu \in[0,1]$ ) between the mobile target and $n_{j}$ within finite steps can be described as

$$
\mu_{\tau}\left(n_{j}\right)=\operatorname{Pr}\left[\min \left(d_{j}(i)\right) \leqslant R \mid i \leqslant \tau\right]
$$

where $\tau \in \mathbb{N}$ denotes a bound of step number, and $\mu_{\tau}$ denotes a function of certainty degree which is dependent on $d_{j}(i)$ given $\tau$.

Taking the equation (8) as the membership function, a fuzzy set of the stationary nodes is defined as

$$
\mathcal{N}_{\tau, \eta}=\left\{n_{j} \mid \mu_{\tau}\left(n_{j}\right) \geqslant \eta\right\}
$$

where $\eta \in[0,1]$ denote a threshold of certainty degree and $\mathcal{N}_{\tau, \eta}$ denotes the subset of stationary nodes considered to be along the movement path of the mobile target given $\tau$ and $\eta$.

To calculate $d_{j}(i)$, a polar coordinate system is considered where $r$ denotes the relative distance from the original position of the mobile target, and $\varphi$ denotes the relative angle measured with respect to the initial movement direction of the mobile target. The localization and tracking techniques can be referred from works such as [33]. Under such coordinate system, the position coordinates of the mobile target after $i$ steps (denoted by $\vec{e}_{i}$ ) and the position coordinates of $n_{j}$ (denoted by $\vec{e}_{j}$ ) can be respectively described as

$$
\begin{aligned}
\vec{e}_{i} & =\left(r_{i}, \varphi_{i}\right) \\
\vec{e}_{j} & =\left(r_{j}, \varphi_{j}\right)
\end{aligned}
$$

According to the law of cosines in trigonometry,

$$
\begin{aligned}
& d_{j}(i)=\left|\vec{e}_{i}-\vec{e}_{j}\right| \\
& =\left|\vec{e}_{i}\right|^{2}+\left|\vec{e}_{j}\right|^{2}-2\left|\vec{e}_{i}\right|\left|\vec{e}_{j}\right| \cdot \cos \left\langle\vec{e}_{i}, \vec{e}_{j}\right\rangle \\
& =r_{i}^{2}+r_{j}^{2}-2 r_{i} r_{j} \cdot \cos \left(\varphi_{i}-\varphi_{j}\right)
\end{aligned}
$$

The $r_{j}$ and $\varphi_{j}$ are known constants given the original position and the initial movement direction of the mobile target. However, due to the random turning angle as described in equation (3), $\vec{e}_{i}$ is a stochastic process as $\left\{\left(r_{i}, \varphi_{i}\right): i \in \mathbb{N}\right\}$, where $r_{i}$ and $\varphi_{i}$ are random variables representing the displacement distance and angle observed after $i$ steps, respectively. To quantify the contact potential, this movement process is further analysed in the Section IV.

\section{Strategy Model (Design of Forward-Wait-Deliver)}

Based on the predictable contact, strategies can be made for efficient forwarding. Let $m_{k}$ denotes the task of delivering a data item from the gateway node to a mobile destination node, and a whole set of tasks is denoted by $\mathcal{M}$. In this paper, we assume these delivery tasks are independent, which represents a generalised scenario that can be either a delivery to a whole group of destinations or separate deliveries to a single destination. The strategy of relay sharing for group delivery, 
as in [10], is left for future work. Considering delivery tasks of different importance is also beyond the scope of this paper.

For the delivery with delay tolerance, predictable contact can be considered in order to reduce the number of transmissions. Let $B_{k}$ denote the delay budget of $m_{k}$, then corresponding maximum allowed step number (denoted by $\tau_{k}$ ) can be calculated as

$$
\tau_{k}=\left\lfloor\frac{B_{k}}{T}\right\rfloor
$$

Thus, for example, if the delay budget is 1300 seconds and the step period is 200 seconds then there are 6 permitted steps.

As with the delivery scheme considered in [10], packets are firstly sent to the selected stationary relay nodes by multihop forwarding for later last-hop delivery. Then the delivery is accomplished by the stationary relay node when the mobile destination node is directly contactable. Based on the fuzzy set defined by the equation (9), qualified candidate stationary nodes for $m_{k}$ can be defined as

$$
\mathcal{N}_{k}(\eta)=\left\{n_{j} \mid \mu_{\tau_{k}}\left(n_{j}\right) \geqslant \eta\right\}
$$

As the stationary nodes form a fixed network topology, we assume knowledge of forwarding hops from the gateway node to any stationary node $n_{j}$ is available at the gateway node, which is denoted by $H_{j}$. For each delivery, the stationary node with the least forwarding hops is selected from the candidates as

$$
h_{k}(\eta)=\min _{n_{j} \in \mathcal{N}_{k}(\eta)}\left(H_{j}\right)
$$

where $h_{k}(\eta)$ denotes the minimum hop number for $m_{k}$ given $\eta$.

Without considering retransmission and duplication issues, transmission consumed energy is primarily proportional to the number of forwarding hops. As the final hop from the relay to the destination should be counted as well, the forwarding cost for $m_{k}$ is defined as

$$
c_{k}(\eta)=1+h_{k}(\eta)
$$

where the plus one counts for the expected last-hop delivery.

As defined by equation (14), any stationary node selected from $\mathcal{N}_{k}(\eta)$ guarantees the certainty degree $\eta$ for on-time contact with the mobile destination. Ideally, a successful contact has the direct contribution to final delivery and therefore the satisfactory degree of delivery is expected as

$$
s_{k}(\eta)=\eta
$$

where $s_{k}(\eta)$ denotes the expected satisfactory degree of delivery given $\eta$.

Finally, the delivery utility is considered as a metric to assess the efficiency of the completion of the whole set of delivery tasks. As the ratio of overall satisfactory degree to the overall forwarding cost, the delivery utility (denoted by $u$ ) is defined as

$$
\begin{aligned}
u(\eta) & =\frac{\sum_{m_{k} \in \mathcal{M}} s_{k}(\eta)}{\sum_{m_{k} \in \mathcal{M}} c_{k}(\eta)}=\frac{M \cdot \eta}{M+\sum_{m_{k} \in \mathcal{M}} h_{k}(\eta)} \\
& =\frac{\eta}{1+h_{\mathrm{avg}}(\eta)} \leqslant \frac{\eta}{1+h_{\min }(\eta)}
\end{aligned}
$$

where $u(\eta)$ denotes the achievable utility given $\eta, M$ denotes the total number of delivery tasks, $h_{\mathrm{avg}}(\eta)$ denotes the average forwarding hops over all the deliveries, and $h_{\min }(\eta)$ denotes the minimum forwarding hops for single delivery.

The utility value reflects the satisfactory degree contributed by each forwarding hop for the whole set of delivery tasks. Equation (18) shows that the delivery utility is dependent on the certainty threshold $\eta$ and is related to the average forwarding hops. Although the direct optimisation of delivery utility is impossible as it requires analysis on a case-by-case basis, equation (18) shows that the maximum of utility value is bounded by the minimum forwarding hops for single delivery. Consequently, it is possible to realise a higher achievable utility by adjusting $\eta$, as a lower threshold of required certainty can provide more candidates and therefore the minimum hop number of forwarding tends to decrease. To explore this possibility, further analysis and simulation are conducted in Section V.

\section{Contact Modelling From Directional MOVEMENT}

\section{A. Directional Correlation}

In Section III, an analytical framework is provided for exploiting mobility in strategic forwarding. Based on the mobility model, the predictable contact with a mobile target can be utilised to select relay(s) from a set of stationary nodes. However, because the movement direction changes randomly in a correlated pattern, it is not easy to obtain a simple expression of the contact potential. To this end, further analysis is required on the stochastic process of movement.

As assumed in equations (1)(2) and (3), the uncertainty of movement results from the Random Turning Angle (RTA) between two successive steps. In Figure 3, a comparison is shown between three types of distribution for RTA. Traditionally, it is assumed that the current movement direction may change to any other direction with equal probability. This uncorrelated change can be represented by an isotropic uniform distribution. By restricting the maximum allowed direction change, the bounded uniform distribution [27] leads to correlation in the step direction. However, this bounded uniform distribution fails to reflect the natural phenomenon that smaller deviations tend to have higher frequency of occurrence. Therefore, in this paper, the truncated Normal distribution is proposed as the probability model for RTA.

Based on the zero-centred Normal distribution with symmetric truncation, the probability density of the RTA is given as

$$
\operatorname{Pr}[\Theta=\delta]=\frac{\phi\left(\frac{\delta}{\sigma}\right)}{\sigma\left(2 \Phi\left(\frac{\epsilon}{\sigma}\right)-1\right)}
$$

where $\phi$ and $\Phi$ denotes the probability density function and cumulative distribution function of the standard Normal distribution, respectively. $\sigma>0$ denotes the standard deviation, $\epsilon \in[0, \pi]$ denotes the truncation bound, and $\Theta$ denotes the random turning angle as defined in equation (3).

Then the probability of a turning angle within a given 


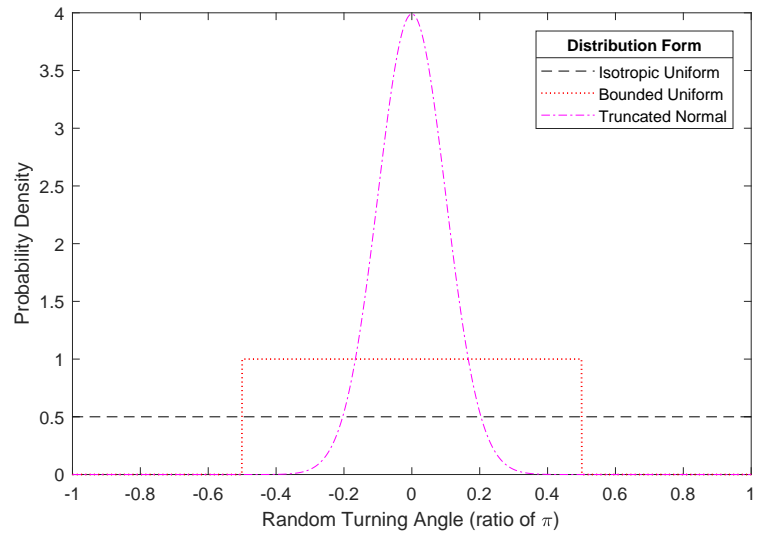

Fig. 3. A Comparison between Three Types of Probability Distribution

interval can be expressed as

$$
\operatorname{Pr}\left[l_{1} \leqslant \Theta \leqslant l_{2}\right]=\frac{\Phi\left(\frac{l_{2}}{\sigma}\right)-\Phi\left(\frac{l_{1}}{\sigma}\right)}{2 \Phi\left(\frac{\epsilon}{\sigma}\right)-1}
$$

where $-\pi \leqslant l_{1}<l_{2} \leqslant \pi$ denote the two ends of the interval.

Due to the truncation, the probability is zero outside the bounded range, which can be proved as

$$
\begin{aligned}
\operatorname{Pr}[\epsilon<|\Theta| \leqslant \pi] & =1-\operatorname{Pr}[-\epsilon \leqslant \Theta \leqslant \epsilon] \\
& =1-\frac{\Phi\left(\frac{\epsilon}{\sigma}\right)-\Phi\left(\frac{-\epsilon}{\sigma}\right)}{2 \Phi\left(\frac{\epsilon}{\sigma}\right)-1} \\
& =1-\frac{\Phi\left(\frac{\epsilon}{\sigma}\right)-\left(1-\Phi\left(\frac{\epsilon}{\sigma}\right)\right)}{2 \Phi\left(\frac{\epsilon}{\sigma}\right)-1} \\
& =0
\end{aligned}
$$

Meanwhile, the effect of truncation depends on the relative relationship between $\sigma$ and $\epsilon$. As shown in Figure 4, the possible turning angle is represented as a random variable having a continuous distribution between truncation bounds. Given a certain truncation bound $\epsilon$, the shape of distribution curve varies with the value of $\sigma$. With an increasing $\sigma$ value, the density difference within the bounded range becomes smaller. For the special case that $\sigma$ is extremely large, a uniform distribution is approximated.

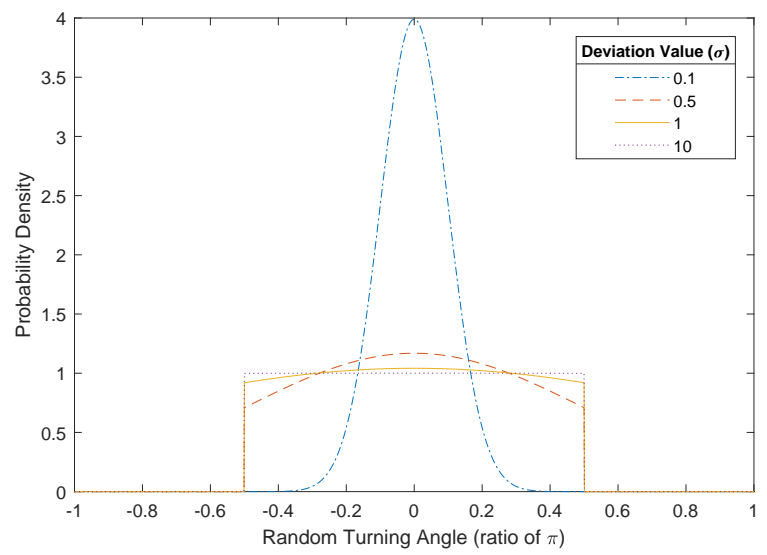

Fig. 4. Effect of Deviation Value on the Truncated Normal Distribution
When the value of $\sigma$ is sufficiently small compared with the truncation bound $\epsilon$, the truncated Normal distribution is very close to a Normal distribution without truncation. To measure the relationship between $\sigma$ and $\epsilon$, we define a ratio as

$$
\rho=\frac{\epsilon}{\sigma}
$$

Then equation (19) can be written as

$$
\begin{aligned}
\operatorname{Pr}[\Theta=\delta] & =\frac{\phi\left(\frac{\delta}{\sigma}\right)}{\sigma(2 \Phi(\rho)-1)} \\
& \approx \frac{\phi\left(\frac{\delta}{\sigma}\right)}{\sigma} \quad \rho \geqslant \rho_{\mathrm{th}}
\end{aligned}
$$

where $\rho_{\text {th }}$ is a threshold value that satisfies $\Phi(\rho) \approx 1$ and typically $\rho_{\text {th }} \geqslant 4$.

With the condition $\rho \geqslant \rho_{\text {th }}$, the truncated Normal distribution can be simplified such that it is only affected by the single parameter $\sigma$ which stands for the Standard Deviation of the Turning Angles (SDTA) in this paper. In other words, the Normal distribution is considered naturally bounded within a certain range. To apply this single-parameter description, the maximum allowed value of $\sigma$ is given as

$$
\sigma_{\max }=\frac{\epsilon_{\max }}{\sigma_{\min }}=\frac{\pi}{\rho_{\mathrm{th}}}
$$

\section{B. Movement Estimation}

As described in Section III, the prediction of contact is dependent on the estimation of movement, which the key of strategy making. Here, the stochastic properties are further analysed to estimate the movement.

Based on equation (4) and (5), the displacement distance projected on the axial direction can be given as

$$
\begin{aligned}
r_{i} \cdot \sin \varphi_{i} & =\sum_{\zeta=1}^{i}\left(\lambda_{i} \cdot \sin \omega_{i}\right) \\
& =V \cdot T \cdot \sum_{\zeta=1}^{i}\left(\sin \omega_{\zeta}\right)
\end{aligned}
$$

Similarly, the displacement distance projected on the radial direction can be given as

$$
r_{i} \cdot \cos \varphi_{i}=V \cdot T \cdot \sum_{\zeta=1}^{i}\left(\cos \omega_{\zeta}\right)
$$

Dividing equation (25) by equation (26), we obtain

$$
\tan \varphi_{i}=\frac{\sum_{\zeta=1}^{i}\left(\sin \omega_{\zeta}\right)}{\sum_{\zeta=1}^{i}\left(\cos \omega_{\zeta}\right)}
$$

Consequently,

$$
\varphi_{i}=\arctan \left(\frac{\sum_{\zeta=1}^{i}\left(\sin \left(\theta_{0}+\sum_{\xi=1}^{\zeta} \Theta_{\xi}\right)\right)}{\sum_{\zeta=1}^{i}\left(\cos \left(\theta_{0}+\sum_{\xi=1}^{\zeta} \Theta_{\xi}\right)\right)}\right)
$$


where $\varphi_{i}$ denotes the displacement angle after $i$ steps.

Due to the complexity of equation (28), the probability distribution of $\varphi_{i}$ cannot be solved directly. However, according to the central limit theorem [34], independent and identically distributed (i.i.d.) random variables tend towards the Normal distribution. Therefore, the distribution of the displacement angle after a number of steps is expected to be a bell-shape curve given as

$$
f_{\text {Gauss }}(z)=\alpha \cdot \exp \left(-\left(\frac{z-\beta}{\gamma}\right)^{2}\right)
$$

where $f_{\text {Gauss }}$ denotes the Gaussian function, $z$ denotes the independent variable, $\alpha$ denotes the amplitude factor, $\beta$ denotes the centroid factor, and $\gamma$ denotes the scale factor.

TABLE III

SynthetiC TraCES Generation FOR MONTE CARlo EXPERIMENTS

\begin{tabular}{|c|c|}
\hline Parameters & Values \\
\hline Truncation Bound & $\pm \pi$ \\
\hline$\rho_{t h}$ & 4 \\
\hline$\sigma$ (SDTA) & $5,10,15,20,25,30,35,40,45$ (degrees) \\
\hline Movement Speed & $1 \mathrm{~m} / \mathrm{s}$ \\
\hline Initial Direction & x-axis positive direction \\
\hline Original Position & central point $(0,0)$ \\
\hline Step Period & 30 seconds \\
\hline Observation Period & 3600 seconds \\
\hline Number of Traces & 10000 for each SDTA value \\
\hline
\end{tabular}

Based on the Monte Carlo method [35], simulation experiments are conducted to confirm the validity of distribution and to obtain the model parameters. Given initial parameters ${ }^{3}$ (specified in Table III), a set of synthetic traces can be generated as shown in Figure 5. A clearer view can be provided by sampling the trace points only at the selected steps as shown in Figure 6.

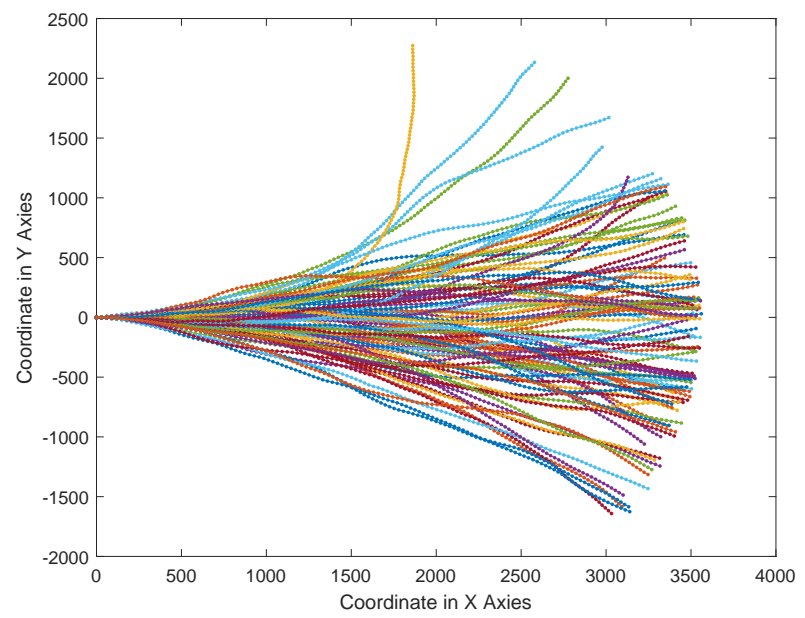

Fig. 5. 100 Traces Generated by Setting SDTA to 10 Degrees

As shown in Figure 7, the frequency of a particular displacement angle is counted at each sampled step. The statistical results visually show a trend towards the Normal distribution. By fitting the simulated frequency distribution of each

\footnotetext{
${ }^{3}$ settings in this paper are based on the normal walking behaviours but scalable to other scenarios
}

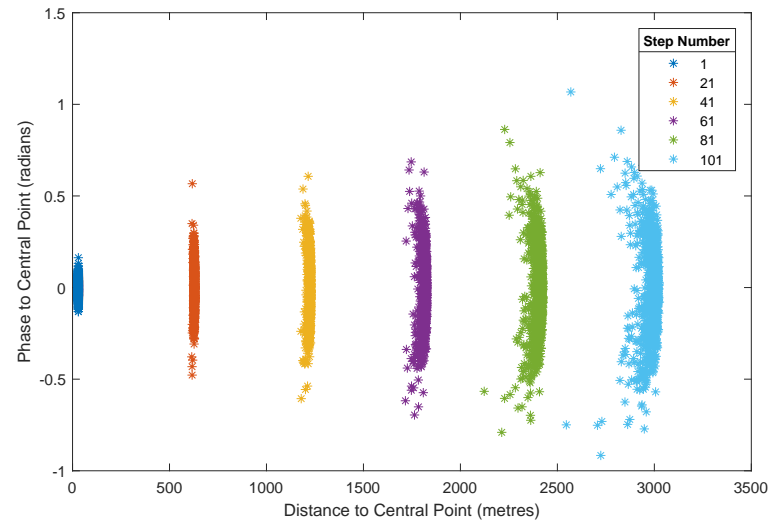

Fig. 6. Trace Points Sampled at Selected Steps from 1000 Traces (Setting SDTA to 10 Degrees)

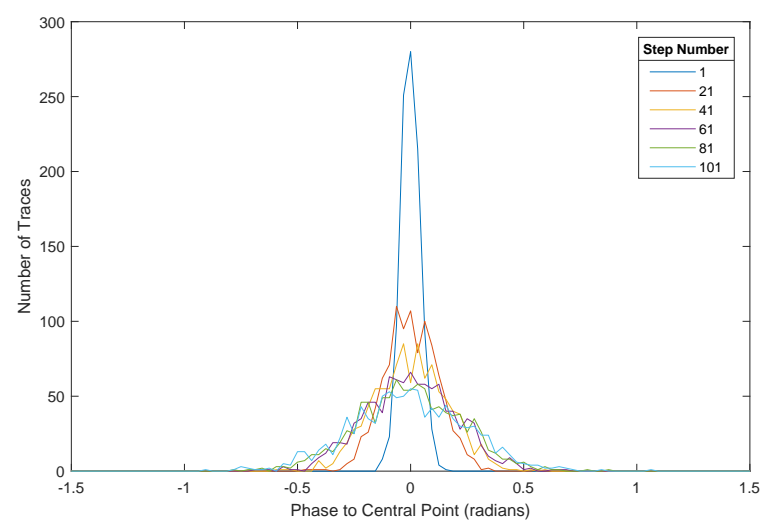

Fig. 7. Frequency Statistics of Displacement Angle Sampled at Selected Steps from 1000 Traces (Setting SDTA to 10 Degrees)

sampled step to the bell-shape curve given in (29), functional relationships are reflected between the three model parameters and the step numbers. As shown in Figure 8, the amplitude factor decreases and the scale factor increases non-linearly with increasing step number, whilst the centroid factor remains constant. With the 'NonlinearLeastSquares' fitting method and 'Trust-Region' fitting algorithm provided by the MATLAB Curve Fitting Toolbox, the factor relationships are obtained as

$$
\left\{\begin{array}{l}
\alpha=a_{1} \cdot i^{a_{2}}+a_{3} \\
\beta=0 \\
\gamma=b_{1} \cdot i^{b_{2}}+b_{3}
\end{array}\right.
$$

where $\alpha, \beta, \gamma$ are the three factors defined in (29), $a_{1}, a_{2}, a_{3}, b_{1}, b_{2}, b_{3}$ are parametric constants, and $i$ denotes the step number.

As shown in Figure 9, further fitting the parameters obtained in (30), the relationships can be found with the SDTA. Using the same fitting technique, the parameter relationships are 


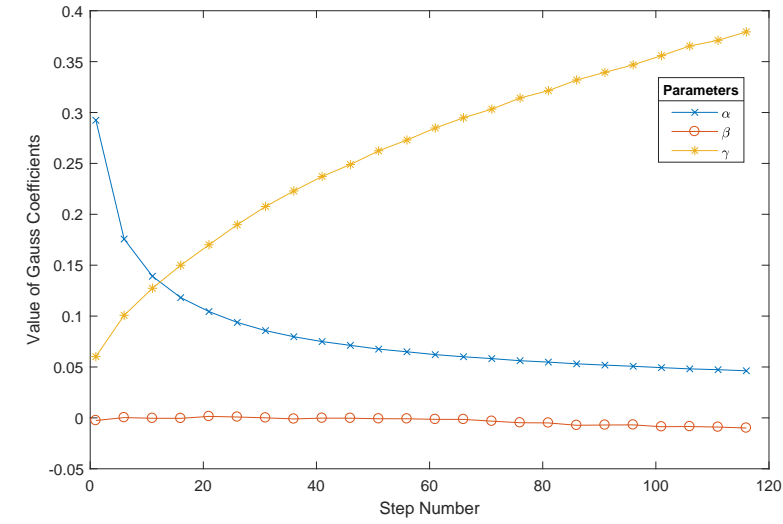

Fig. 8. Fitted Model Parameters at Different Step Numbers (Setting SDTA to 10 Degrees)

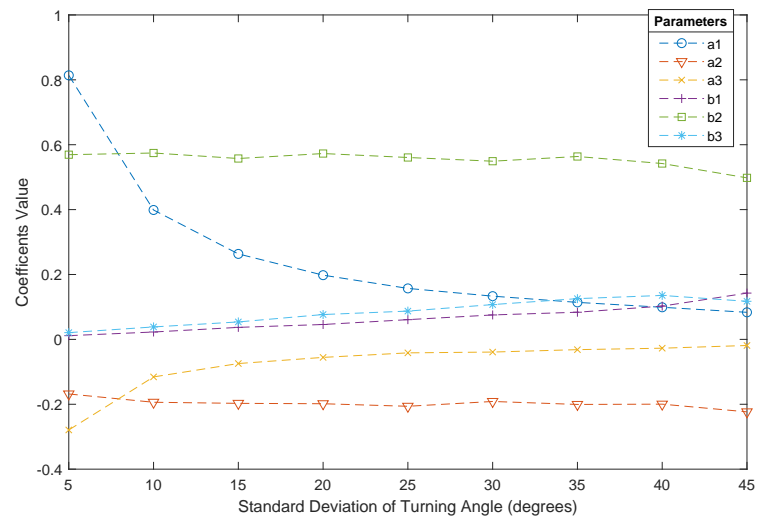

Fig. 9. Further Fitted Model Parameters at Different SDTA Values

obtained as

$$
\left\{\begin{array}{l}
a_{1}=p_{1} \cdot \sigma^{p_{2}} \\
a_{2}=p_{3} \\
a_{3}=p_{4} \cdot \sigma^{p_{5}} \\
b_{1}=p_{6} \cdot \sigma+p_{7} \\
b_{2}=p_{8} \\
b_{3}=p_{9} \cdot \sigma+p_{10}
\end{array}\right.
$$

where $a_{1}, a_{2}, a_{3}, b_{1}, b_{2}, b_{3}$ are parametric constants defined in (30), $p_{1}$ to $p_{10}$ are fitting parameters, and $\sigma$ denotes the SDTA.

Based on equations (29)(30) and (31), the curve shape can be defined as a parametric model

$$
\left\{\begin{array}{l}
f_{\text {shape }}(z, \sigma, i)=\alpha(\sigma, i) \cdot \exp \left(-\left(\frac{z-\beta}{\gamma(\sigma, i)}\right)^{2}\right) \\
\alpha(\sigma, i)=\left(p_{1} \cdot i^{p_{2}}\right) \cdot \sigma^{p_{3}}+\left(p_{4} \cdot i^{p_{5}}\right) \\
\gamma(\sigma, i)=\left(p_{6} \cdot i+p_{7}\right) \cdot \sigma^{p_{8}}+\left(p_{9} \cdot i+p_{10}\right) \\
\beta=0
\end{array}\right.
$$

where $f_{\text {shape }}$ denotes the curve shape function, $z$ denotes the independent variable, $\sigma$ denotes the SDTA, $i$ denotes the step number, and $p_{1}$ to $p_{10}$ are parameters (listed in Table IV) solved under Table III configurations.

Then the probability distribution function of displacement angle after certain steps (denoted by $f_{\text {pdf }}$ ) can be obtained as

$$
\operatorname{Pr}\left[\varphi_{i}=\delta\right]=f_{\mathrm{pdf}}(\delta, \sigma, i)=\frac{f_{\text {shape }}(\delta, \sigma, i)}{\int_{-\pi}^{\pi} f_{\text {shape }}(z, \sigma, i) d z}
$$

As shown in equation (33), the probability distribution of displacement angle varies over steps and is dependent on SDTA. This distribution model provides a tool to quantify the directional movement, which will be applied in Section IV-C for contact prediction.

TABLE IV

SOlVed Model Parameters

\begin{tabular}{|c|c||c|c|}
\hline Parameters & Values & Parameters & Values \\
\hline$p_{1}$ & 0.0675 & $p_{6}$ & 0.1694 \\
\hline$p_{2}$ & -1.0207 & $p_{7}$ & -0.0092 \\
\hline$p_{3}$ & -0.1976 & $p_{8}$ & 0.5542 \\
\hline$p_{4}$ & -0.0157 & $p_{9}$ & 0.1627 \\
\hline$p_{5}$ & -1.1786 & $p_{10}$ & 0.0137 \\
\hline
\end{tabular}

\section{Contact Prediction}

In Section IV-B, a parametric model is proposed to estimate the displacement angle over steps based on the Standard Deviation of the Turning Angles (SDTA). Now we apply this estimation model in an three-phases approach to describe the contact certainty based on the relative distance (denoted by $r$ ) from the original position of the mobile target, and the relative angle (denoted by $\varphi$ ) measured with respect to the initial movement direction of the mobile target.

For the first phase, an estimated step number (denoted by $\left.i_{\text {est }}\right)$ is calculated as

$$
i_{\mathrm{est}}=\frac{r}{V \cdot T}
$$

where $V$ denotes the average speed and $T$ denotes the step period.

For the second phase, the range of directional coverage is given as

$$
\begin{gathered}
{\left[\varphi_{\min }^{\text {cover }}, \varphi_{\max }^{\text {cover }}\right]} \\
\varphi_{\min }^{\text {cover }}=\varphi-\sin \left(\frac{R}{r}\right) \\
\varphi_{\max }^{\text {cover }}=\varphi+\sin \left(\frac{R}{r}\right)
\end{gathered}
$$

where $\varphi_{\min }^{\text {cover }}, \varphi_{\max }^{\text {cover }} \in[-\pi, \pi]$ denotes the minimum/maximum coverage angle and $R$ denotes the radius of contact range.

For the third phase, the contact certainty (denoted by $\mu_{\text {est }}$ ) is finally described as

$$
\begin{aligned}
\mu_{\mathrm{est}} & =\operatorname{Pr}\left[\varphi_{\max }^{\mathrm{cover}} \geqslant \varphi_{i_{\mathrm{est}}} \geqslant \varphi_{\mathrm{min}}^{\mathrm{cover}}\right] \\
& =\int_{\varphi_{\min }^{\text {cover }}}^{\varphi_{\max }^{\text {cove }}} f_{\mathrm{pdf}}\left(\delta, \sigma, i_{\mathrm{est}}\right) d \delta \\
& =f_{\sigma}(r, \varphi)
\end{aligned}
$$

where $f_{\sigma}$ denotes the estimation function given $\sigma$.

Thus, the contact certainty is formulated as a spatial distribution given SDTA. This distribution model describes statistically predicable contact with spatial points, which is later applied for the selection of stationary relay node. With 


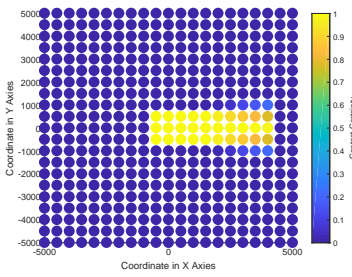

(a) 5 Degrees

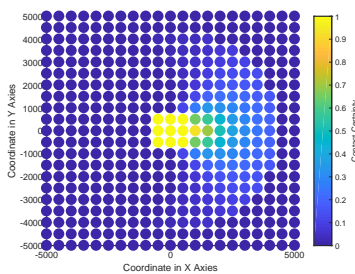

(e) 25 Degrees

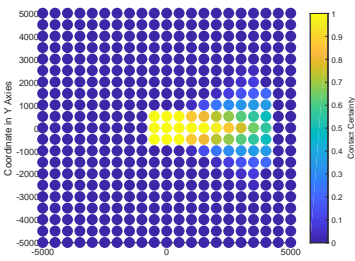

(b) 10 Degrees

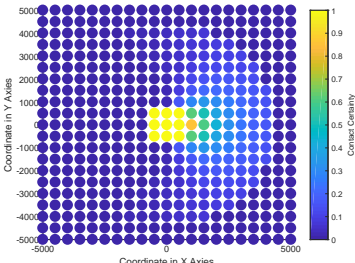

(f) 30 Degrees

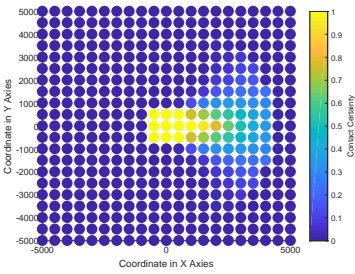

(c) 15 Degrees

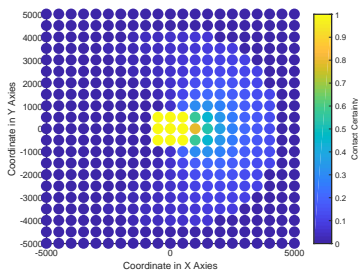

(g) 35 Degrees

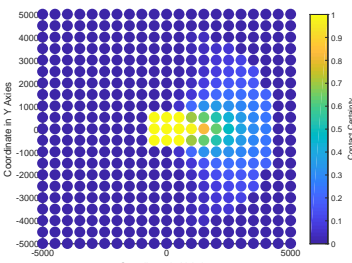

(d) 20 Degrees

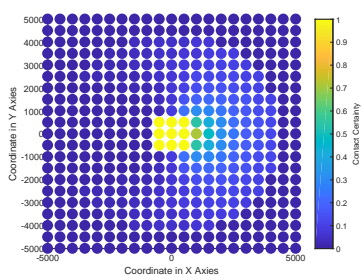

(h) 40 Degrees

Fig. 10. Spatial Distribution of Contact Certainty Based on the Estimation Model for Different Values of SDTA

configurations specified by Table $\mathrm{V}$, a simulation is firstly conducted to visually demonstrate this distribution model.

As shown in Figure 10, the geographical region is equally divided into multiple grids, where the mobile node is originally located at the central grid point with initial movement direction towards the $\mathrm{x}$-axis positive direction. Based on the equation (38), the contact certainty is estimated for each grid point given SDTA. The results show that this model can reflect the decreasing predictability with increasing value of SDTA (i.e. the less directional correlation). The varying predictability does not affect the central nine grid points because they are directly contactable and no estimation is required.

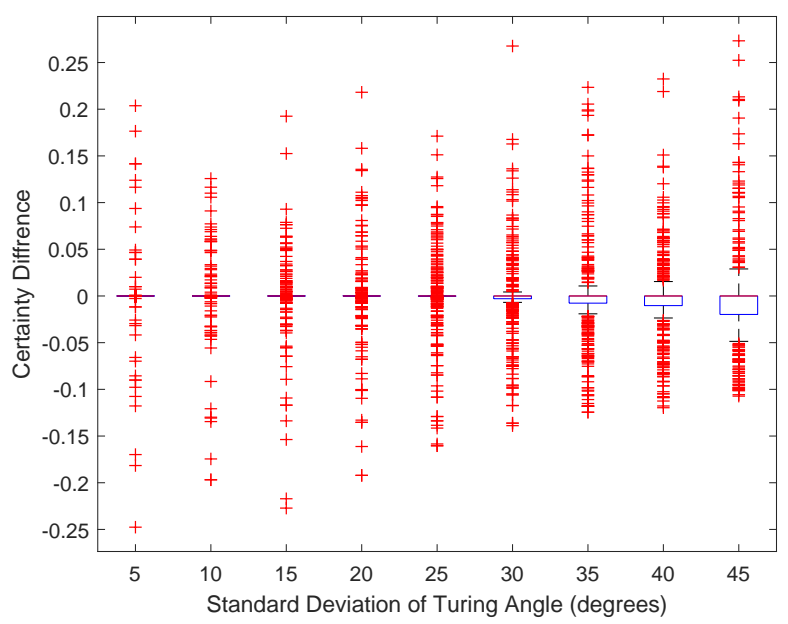

Fig. 11. Box Plot of Certainty Difference for the Accuracy Validation

To further validate the model accuracy, test traces are generated synthetically (100 traces for each SDTA value with same parameters listed in Table III). The ratio of contactable traces is calculated for each grid point as the tested contact certainty. The certainty difference is defined as the tested certainty minus the estimated certainty, so a positive difference value reveals an underestimate and a negative difference value reveals an overestimate. In Figure 11, the certainty difference at each grid points is shown for different SDTA values. Because equation (38) is not an accurate calculation of contact probability, either underestimation or overestimation may exist as the results show. However, it can be observed the model can adaptively react to the varying SDTA so that the difference values are mostly bounded by 0.1 and the rare extreme values are roughly bounded by 0.25 . As a conclusion, the approximate estimation model can reflect the effect of directional correlation on contact certainty.

TABLE V

Simulation CONFIGURATIONS FOR CONTACT PREDiCTION

\begin{tabular}{|c|c|}
\hline Parameters & Values \\
\hline Mobility Configurations & Same with Table III \\
\hline Model Parameters & Same with Table IV \\
\hline Region Size & $10000 * 10000$ metres \\
\hline Grid Size & $500 * 500$ metres \\
\hline Radius of Contact Range & 750 metres \\
\hline
\end{tabular}

\section{Contact Modelling For Relay Selection}

In Section III, a mathematical analysis on strategy model reveals the possibility of higher achievable utility by adjusting the threshold of contact certainty in stationary relay node selection. Now we further explore its practical effect in a simulation scenario (configurations specified by Table VI).

As shown in Figure 12, a rectangular region is considered as the network area where a certain number of stationary nodes (denoted by blue squares) are uniformly deployed. Single-hop connectivities (denoted by blue dashed lines) exist between neighbouring stationary nodes, which form a grid network topology. One of the stationary nodes (located at the bottom right corner in our case) serves as the gateway node which connects to the outside of network area. A certain number of delivery tasks are to be accomplished, where all the deliveries come from the gateway node and each goes to a mobile destination node. The original position (denoted by a blue circle) and initial movement direction (denoted by a blue solid line) of the mobile destination node are randomly and independently decided for each delivery task.

To accomplish a delivery task, one stationary node is selected from a set of candidates (denoted by black dots) as 


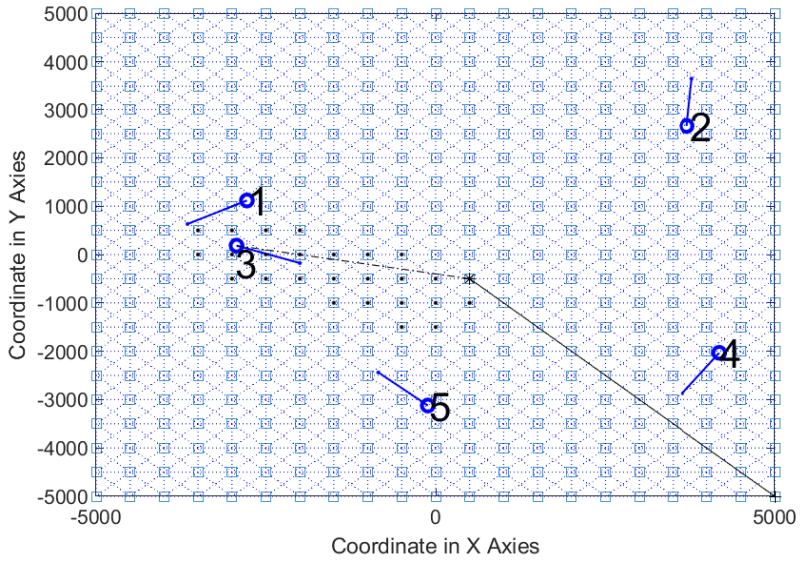

Fig. 12. Illustrated Delivery Solution for the Third of Five Tasks (Based on the Linear Trajectory Model by Setting Delay Budget to 3000 Seconds)

the last-hop relay. The selected stationary relay node (denoted by a black asterisk) is responsible for the direct delivery when the mobile destination node is contactable. Based on the network topology formed by stationary nodes, the gateway node connects to the selected stationary relay node by a multihop forwarding route. The position-based unicast routing is considered for packet forwarding due to its low computation and communication overhead [36]. In Figure 12, the black solid line shows the multi-hop forwarding route and the black dash-dot line indicates the expected contact with the mobile destination node. By selecting the stationary node with the fewest possible forwarding hops as the relay node, the transmission times can be reduced which is beneficial for prolonging network lifetime. Thus, the remaining problem is listing candidates from stationary nodes based on the mobility information.

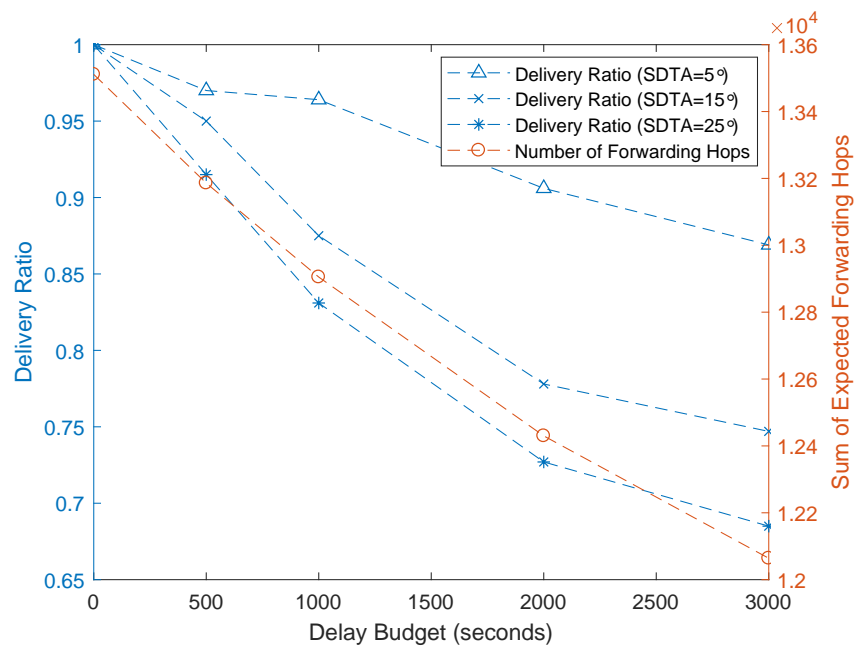

Fig. 13. Effect of Delay Budget on Linear Trajectory Model

In Figure 13 and 14, we show the limitation of a linear trajectory model and how our proposed fuzzy path model overcomes it. To evaluate the performance of relay selection, synthetic traces are generated (one trace for each delivery task)

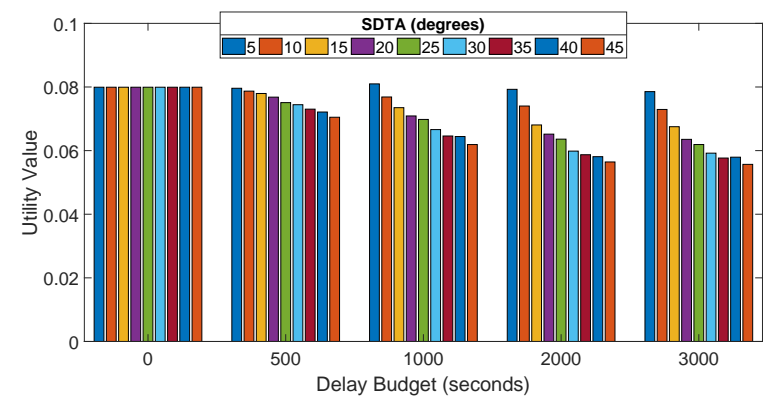

(a) Linear Trajectory Model

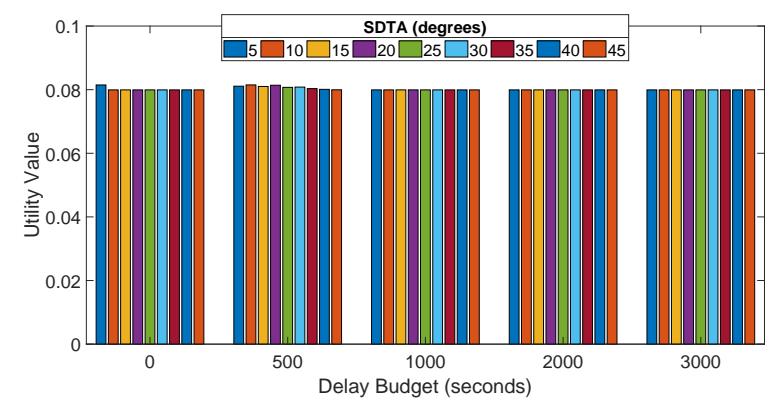

(b) Fuzzy Path Model

Fig. 14. Comparison of Delivery Utility for Different Values of Delay Budget and SDTA

and the trace generation is repeated for different SDTA values (referring Table III). For delivery without delay tolerance (i.e. delay budget equals to zero in Figure 13), the stationary relay node is selected from the immediate neighbouring nodes identified given the latest position of the mobile node. With increasing budget of delivery delay $(500,1000,2000$, and 3000 seconds in Figure 13), more stationary nodes can be listed as candidates by predicting contact based on linear trajectory model and therefore the number of forwarding hop can be reduced. However, the delivery ratio $^{4}$ will decrease at the same time, which worsens with higher SDTA because of the lower persistence of directional movement. Consequently, the delivery utility (as defined in equation (18)) will decrease with increasing SDTA especially when the delay budget is large, which is shown by Figure 14(a). In contrast, the achievable utility is nearly constant over varying SDTA even for a large delay budget (as shown in Figure 14(b)), because proposed fuzzy path model can actively adapt to different degrees of directional correlation.

In Figure 15, more details of the comparison are given for a relative large delay budget (3000 seconds in this case). For the scheme without prediction, only immediate neighbouring nodes can be selected as the relay and therefore the utility is constant and does not change with the SDTA. For the scheme based on linear trajectory model, the utility decreases with increasing SDTA due to lower delivery ratio caused by more randomness in movement. Because of the large delay budget, the linear trajectory model is no longer accurate for contact prediction so that the achieved utility is always worse than the scheme without prediction. With the proposed fuzzy path

\footnotetext{
${ }^{4}$ the percentage of delivery tasks which are accomplished within delay budget
} 


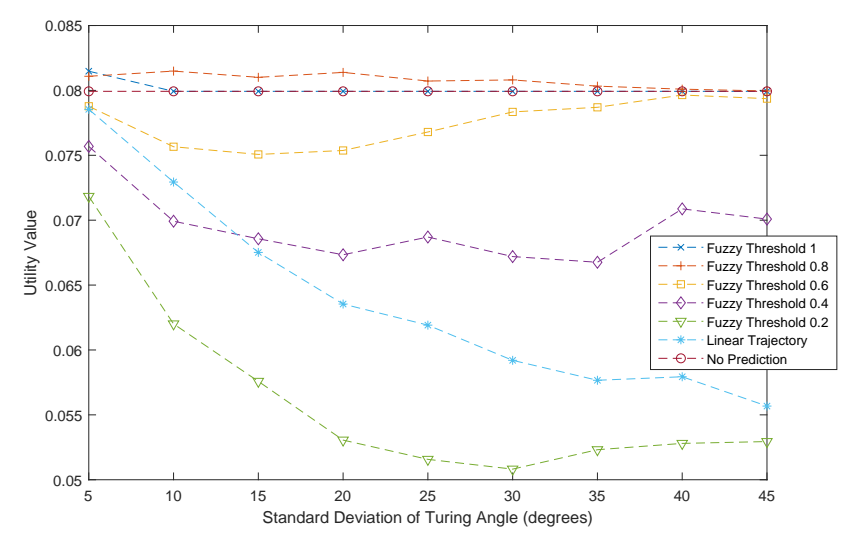

Fig. 15. Comparison of Delivery Utility for Different Values of SDTA (Setting Delay Budget to 3000 Seconds)

model, higher achievable utility can be realised because of its dynamic reaction to the movement directivity.

As shown in Figure 15, the delivery utility achieved by the proposed fuzzy path model is related to the threshold ${ }^{5}$ of certainty degree (i.e. the minimum required contact certainty to qualify a set of stationary nodes as relay candidates). With increasing certainty threshold, the utility achieved by the fuzzy path model approximates the scheme without prediction, due to lower allowed uncertainty. By filtering out unqualified candidates (i.e. stationary nodes who do not have enough contact certainty), the proposed fuzzy path model can always outperform the linear trajectory model when the threshold value is no less than 0.6 . For the performance at the threshold value 0.6 , the delivery utility increases after a certain SDTA value (15 degrees in this case) because there is no more opportunistic forwarding when the movement becomes less predictable. With a higher threshold (no less than 0.8 in this case), its performance is always no worse than the scheme without prediction, owing to the relay selection from a more appropriate set of candidates.

Compared with the zero allowance of uncertainty (i.e. a threshold value of 1), a moderate uncertainty threshold (i.e. a value 0.8 in this case) can achieve higher utility because it tends to identify better relay candidates (who have fewer forwarding hops whilst keeping a low enough risk of delivery failure). Even when the threshold value equals to one, the utility achieved by the proposed scheme can be higher than the scheme without prediction when the SDTA is very small (5 degrees in this case), as the directivity of movement is strong enough to provide additional candidates without concerning uncertainty. Furthermore, although a higher achievable utility becomes less possible with increasing randomness (as the risk associated with opportunistic delivery increases), it is shown that the mobility with a moderate value of SDTA (less then 30 degrees in this case) is exploitable as directional movement to facilitate packet forwarding.

\footnotetext{
${ }^{5}$ by this we mean a lower-bound degree value
}

TABLE VI

Simulation Parameters For Node SELECTION

\begin{tabular}{|c|c|}
\hline Parameters & Values \\
\hline Network Area & $10000^{*} 10000$ metres \\
\hline Transmission Range & 750 metres \\
\hline Number of Stationary Nodes & $21^{*} 21$ \\
\hline Deployment of Stationary Nodes & uniformly distributed \\
\hline Gateway Location & bottom right corner \\
\hline Routing Algorithm & position-based greedy \\
\hline Original Position & random within network area \\
\hline Initial Direction & random within $[-\pi, \pi]$ \\
\hline Number of Delivery Tasks & 1000 \\
\hline
\end{tabular}

\section{CONCLUSIONS}

In this paper, the feasible exploitation of directional movement in path-unconstrained mobility is investigated for strategic forwarding. The state-of-the-art forwarding schemes are studied and the related literature is reviewed. The main contribution of our research is a systematic framework for analytical modelling of relay selection. Considering current limitations, a novel fuzzy path model is proposed for movement estimation, where the influence of directional correlation is quantified by the standard deviation of the turning angles. Our proposal advances the state-of-the-art because the directional correlation of destination movement is considered to dynamically exploit the node mobility for the optimal selection of the stationary relay node. Simulation results show that higher delivery utility can be achieved by the proposed fuzzy path model, compared with a forwarding scheme without contact prediction or one based on linear trajectory prediction. As a conclusion, the directional movement is exploitable for the dissemination of delay-tolerant information especially when moderate uncertainty is allowed. Further research can be conducted to devise appropriate forwarding strategies in different scenarios.

\section{REFERENCES}

[1] A. Cerpa, J. Elson, D. Estrin, L. Girod, M. Hamilton, and J. Zhao, "Habitat monitoring: Application driver for wireless communications technology," ACM SIGCOMM Computer Communication Review, vol. 31, no. 2 supplement, pp. 20-41, 2001.

[2] J. Ahn, A. Mysore, K. Zybko, C. Krumm, S. Thokala, X. Xing, M. Lian, R. Han, S. Mishra, and T. Hobbs, "Wildsense: Monitoring interactions among wild deer in harsh outdoor environments using a delay-tolerant wsn," Journal of Sensors, vol. 2016, 2016.

[3] A. Mainwaring, D. Culler, J. Polastre, R. Szewczyk, and J. Anderson, "Wireless sensor networks for habitat monitoring," in Proceedings of the 1st ACM International Workshop on Wireless Sensor Networks and Applications, ser. WSNA '02. New York, NY, USA: ACM, 2002, pp. 88-97.

[4] I. F. Akyildiz, D. Pompili, and T. Melodia, "Underwater acoustic sensor networks: research challenges," Ad hoc networks, vol. 3, no. 3, pp. 257$279,2005$.

[5] I. F. Akyildiz and I. H. Kasimoglu, "Wireless sensor and actor networks: research challenges," Ad hoc networks, vol. 2, no. 4, pp. 351-367, 2004.

[6] B. Liu, T. Jiang, Z. Wang, and Y. cao, "Object-oriented network: A named-data architecture toward the future internet," IEEE Internet of Things Journal, vol. 4, no. 4, pp. 957-967, Aug 2017.

[7] Y. Cao, T. Jiang, and Z. Han, "A survey of emerging $\mathrm{m} 2 \mathrm{~m}$ systems: Context, task, and objective," IEEE Internet of Things Journal, vol. 3, no. 6, pp. 1246-1258, Dec 2016.

[8] L. Gou, G. Zhang, D. Bian, W. Zhang, and Z. Xie, "Data dissemination in wireless sensor networks with instantly decodable network coding," Journal of Communications and Networks, vol. 18, no. 5, pp. 846-856, October 2016.

[9] M. T. Nuruzzaman and H. W. Ferng, "A low energy consumption routing protocol for mobile sensor networks with a path-constrained mobile sink," in 2016 IEEE International Conference on Communications (ICC), May 2016, pp. 1-6. 
[10] G. L. Chiou, S. R. Yang, and W. T. Yen, "On trajectory-based i2v group message delivery over vehicular ad-hoc networks," IEEE Transactions on Vehicular Technology, vol. 65, no. 9, pp. 7389-7402, Sept 2016.

[11] R. Kim, H. Lim, and B. Krishnamachari, "Prefetching-based data dissemination in vehicular cloud systems," IEEE Transactions on Vehicular Technology, vol. 65, no. 1, pp. 292-306, Jan 2016.

[12] C.-M. Yu, C.-S. Lu, and S.-Y. Kuo, "Habitual behavior-based opportunistic data forwarding in wildlife tracking," in 2007 4th International Symposium on Wireless Communication Systems. IEEE, 2007, pp. 807808.

[13] C. Liu and J. Wu, "Scalable routing in cyclic mobile networks," IEEE Transactions on Parallel and Distributed Systems, vol. 20, no. 9, pp. 1325-1338, Sept 2009.

[14] Y. Sun, J. Guo, and Y. Yao, "Speed up-greedy perimeter stateless routing protocol for wireless sensor networks (su-gpsr)," in 2017 IEEE 18th International Conference on High Performance Switching and Routing (HPSR), June 2017, pp. 1-6.

[15] J. He, L. Cai, P. Cheng, and J. Pan, "Delay minimization for data dissemination in large-scale vanets with buses and taxis," IEEE Transactions on Mobile Computing, vol. 15, no. 8, pp. 1939-1950, Aug 2016.

[16] Y. Li, D. Jin, P. Hui, and S. Chen, "Contact-aware data replication in roadside unit aided vehicular delay tolerant networks," IEEE Transactions on Mobile Computing, vol. 15, no. 2, pp. 306-321, Feb 2016.

[17] Y. Cao, Z. Sun, N. Wang, M. Riaz, H. Cruickshank, and X. Liu, "Geographic-based spray-and-relay (gsar): An efficient routing scheme for dtns," IEEE Transactions on Vehicular Technology, vol. 64, no. 4, pp. 1548-1564, April 2015.

[18] K. Pandey, S. K. Raina, and R. S. Raw, "Distance and directionbased location aided multi-hop routing protocol for vehicular ad-hoc networks," Int. J. Commun. Netw. Distrib. Syst., vol. 16, no. 1, pp. 7198, Dec. 2016.

[19] X. Wu, K. N. Brown, and C. J. Sreenan, "Data pre-forwarding for opportunistic data collection in wireless sensor networks," ACM Trans. Sen. Netw., vol. 11, no. 1, pp. 8:1-8:33, Jul. 2014.

[20] U. Sadiq and M. Kumar, "Proximol: Proximity and mobility estimation for efficient forwarding in opportunistic networks," in 2011 IEEE Eighth International Conference on Mobile Ad-Hoc and Sensor Systems, Oct 2011, pp. 312-321.

[21] S. Batabyal and P. Bhaumik, "Mobility models, traces and impact of mobility on opportunistic routing algorithms: A survey," IEEE Cоттиnications Surveys Tutorials, vol. 17, no. 3, pp. 1679-1707, thirdquarter 2015.

[22] B. Liang and Z. J. Haas, "Predictive distance-based mobility management for multidimensional pcs networks," IEEE/ACM Transactions on Networking, vol. 11, no. 5, pp. 718-732, Oct 2003.

[23] M. Zhao and W. Wang, "A novel semi-markov smooth mobility model for mobile ad hoc networks." in IEEE Globecom 2006, Nov 2006, pp. $1-5$.

[24] S. Bandyopadhyay, E. J. Coyle, and T. Falck, "Stochastic properties of mobility models in mobile ad hoc networks," IEEE Transactions on Mobile Computing, vol. 6, no. 11, pp. 1218-1229, Nov 2007.

[25] L. Feng, Q. Zhao, and H. Zhang, "Location management based on distance and direction for pcs networks," Comput. Netw., vol. 51, no. 1, pp. 134-152, Jan. 2007.

[26] Q. Zhao, S. C. Liew, S. Zhang, and Y. Yu, "Distance-based location management utilizing initial position for mobile communication networks," IEEE Transactions on Mobile Computing, vol. 15, no. 1, pp. 107-120, Jan 2016.

[27] Z. J. Haas, "A new routing protocol for the reconfigurable wireless networks," in Proceedings of ICUPC 97 - 6th International Conference on Universal Personal Communications, vol. 2, Oct 1997, pp. 562-566 vol.2.

[28] E. A. Codling, M. J. Plank, and S. Benhamou, "Random walk models in biology," Journal of the Royal Society Interface, vol. 5, no. 25, pp. 813-834, 2008.

[29] H.-i. Wu, B.-L. Li, T. A. Springer, and W. H. Neill, "Modelling animal movement as a persistent random walk in two dimensions: expected magnitude of net displacement," Ecological Modelling, vol. 132, no. 1, pp. $115-124,2000$.

[30] J. A. Byers, "Correlated random walk equations of animal dispersal resolved by simulation," Ecology, vol. 82, no. 6, pp. 1680-1690, 2001.

[31] P. Nouvellet, J. P. Bacon, and D. Waxman, "Fundamental insights into the random movement of animals from a single distance related statistic." The American Naturalist, vol. 174, no. 4, pp. 506-514, 2009, pMID: 19737110.

[32] B. N. Clark, C. J. Colbourn, and D. S. Johnson, "Unit disk graphs," Discrete Math., vol. 86, no. 1-3, pp. 165-177, Jan. 1991.
[33] B. Zhou, Q. Chen, and P. Xiao, "The error propagation analysis of the received signal strength-based simultaneous localization and tracking in wireless sensor networks," IEEE Transactions on Information Theory, vol. 63, no. 6, pp. 3983-4007, June 2017.

[34] H. Fischer, A History of the Central Limit Theorem: From Classical to Modern Probability Theory, ser. Sources and Studies in the History of Mathematics and Physical Sciences. Springer New York, 2010.

[35] R. Y. Rubinstein and D. P. Kroese, Simulation and the Monte Carlo method. John Wiley \& Sons, 2016, vol. 10.

[36] K. Sohraby, D. Minoli, and T. Znati, Wireless sensor networks: technology, protocols, and applications. John Wiley \& Sons, 2007.

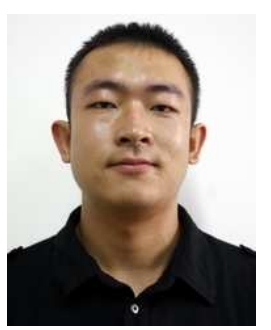

Yuhui Yao received the B.Eng. degree from Beijing University of Posts and Telecommunications (BUPT) in 2014. He is currently a doctoral candidate in the school of Electronic Engineering and Computer Science, Queen Mary University of London (QMUL) in the United Kingdom. His research interests include Software-Defined Networking, Internet of Things, ad hoc networks, and mobility models.

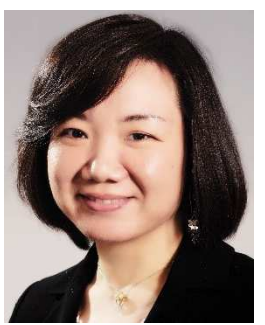

Yan Sun received the B.Eng. degree in Telecommunications Engineering from Beijing University of Posts and Telecommunications in 2001, and the M.Sc. and Ph.D. degrees in Electronic Engineering from Queen Mary University of London in 2003 and 2009, respectively. She joined Siemens Ltd, China, in 2001 as a network optimization engineer and rejoined in 2003 as system engineer in R\&D after obtaining her Master degree in UK. She then worked in Siemens as a product manager for 5 years and returned to Queen Mary as a lecturer in 2009. Her research interests include ad hoc networks, energy saving for modern mobile networks, software defined networks and mobile healthcare networks.

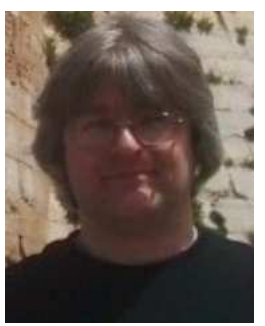

Chris Phillips received the B.Eng. degree in telecommunications engineering in 1987 and the $\mathrm{Ph} . \mathrm{D}$. degree in concurrent discrete event-driven simulation from the Queen Mary University of London, London, U.K. He then worked in industry for nine years as a Hardware and Systems Engineer with Bell Northern Research, Siemens Roke Manor Research, and Nortel Networks. In 2000, he returned to the Queen Mary University of London. A common theme that underpins his research is how management mechanisms can be developed to enable limited resources to be used effectively in a changing or uncertain environment. His work has focused on broadband network protocols, resource management, and resilience.

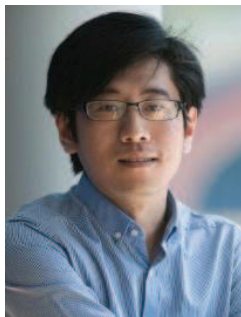

Yue Cao received the Ph.D. degree from the Institute for Communication Systems (ICS), at University of Surrey, Guildford, UK in 2013. He was a Research Fellow at the ICS until September 2016, and Lecturer in Department of Computer and Information Sciences, at Northumbria University, Newcastle upon Tyne, UK until July 2017, and the Senior Lecturer since August 2017. His research interests focus on Intelligent Mobility. $\mathrm{He}$ is the Associate Editor of IEEE Access. 